\title{
Associations of Physical Activity and Sedentary Time with Metabolic Syndrome in Saudi Adult Males
}

This article was published in the following Dove Press journal: Risk Management and Healthcare Policy

\section{Osama Aljuhani (iD) \\ Shaea Alkahtani $\mathbb{D D}^{2}$ \\ Maha Alhussain $\mathbb{D}^{3}$ \\ Lee Smith ${ }^{4}$ \\ Syed Shahid Habib ${ }^{5}$}

'Department of Physical Education, College of Sport Sciences and Physical Activity, King Saud University, Riyadh 4545, Saudi Arabia; ${ }^{2}$ Department of Exercise Physiology, College of Sport Sciences and Physical Activity, King Saud University, Riyadh 4545, Saudi Arabia; ${ }^{3}$ Department of Nutrition and Food Sciences, College of Agricultural and Food Sciences, King Saud University, Riyadh, Saudi Arabia; ${ }^{4}$ The Cambridge Centre for Sport and Exercise Sciences, Anglia Ruskin University, Cambridge CB I IPT, UK; ${ }^{5}$ Department of Physiology, College of Medicine, King Saud University, Riyadh, Saudi Arabia
Correspondence: Osama Aljuhani College of Sport Sciences and Physical Activity, King Saud University, P.O. Box1949, Riyadh II44I, Saudi Arabia Tel +966-556363500

Email oaljuhani@ksu.edu.sa
Purpose: The association between objectively measured sedentary behavior and physical activity with metabolic syndrome (MetS) has been rarely investigated in Saudi Arabia. The purpose of the current study was to examine the association of objectively measured sedentary, light physical activity (LPA), and moderate-vigorous physical activity (MVPA) with MetS among Saudi adult males.

Materials and Methods: The study participants were 103 males from Riyadh, Saudi Arabia (mean age $=54.9 \pm 10.6$ years). Metabolic syndrome was defined based on having three or more of cardiometabolic risk factors. Triaxial accelerometers were used to measure the time spent on sedentary and physical activities across 7 days. A minimum four days with $\geq 10$ hours of wearing time per day were considered a valid data. Binary logistics regression models were performed to examine the association of sedentary and physical activity levels with MetS vs no MetS. Model 1 was unadjusted, models 2, 3, and 4 were mutually controlled for sedentary, light, and MVPA intensities.

Results: About $38 \%$ of males in the present study were classified as having MetS as demonstrated by a significant $(\mathrm{p}<0.05)$ decrease in high-density lipoprotein cholesterol (HDL-C) and a significant $(\mathrm{p}<0.05)$ increase in body weight, body mass index $(\mathrm{BMI})$, waist circumference (WC), systolic blood pressure, glucose, and triglycerides compared to those without MetS. In addition, low levels of LPA (less than 6.3 hours per day) were significantly associated with the risk of having MetS, independent of sedentary and MVPA (odds ratio (OR) 4.26-6.96). The results showed that the associations between sedentary tertiles and MetS were not statistically significant. Levels of MVPA were also not significantly associated with an increased risk of developing MetS in all models.

Conclusion: This study showed that low levels of LPA were significantly associated with the risk of having MetS in Saudi males from Riyadh city, independent of MVPA and sedentary time. The results suggest that future intervention studies should assess the positive effect of increasing levels of LPA in reducing the risk of developing MetS in males.

Keywords: accelerometer, MetS, sedentary time, MVPA, light activity

\section{Introduction}

Metabolic syndrome (MetS) refers to a cluster of three to five cardiometabolic risk factors such as abdominal obesity, high triglycerides (TG), low high-density lipoprotein cholesterol (HDL-C), elevated blood pressure (BP) and elevated fasting blood glucose (FBG). ${ }^{1}$ MetS and its related risk factors have been found to be associated with the risk of developing cardiovascular disease (CVD), coronary heart 
disease (CHD), type-2 diabetes, and all-cause mortality. ${ }^{2,3}$ A recent cross-sectional study showed that about $34.4 \%$ of males in Saudi Arabia have MetS, with this the percentage increasing with age. ${ }^{4}$ The study also showed that the most prevalent MetS components were low levels of HDL-C and abdominal obesity. ${ }^{4}$

Physical activity has been shown to reduce the risk of cardiovascular mortality, ${ }^{5}$ diabetes, ${ }^{6}$ hypertension, ${ }^{7}$ and allcause mortality. ${ }^{8}$ International data indicate that physical activity might also prevent MetS and its associated factors. $^{9-11}$ It has been recommended that adults should accumulate at least 150 minutes of moderate-intensity physical activity (MPA) or 70 minutes of vigorous-intensity physical activity (VPA) weekly. ${ }^{12}$ Data from a systemic review indicated that Saudi males are insufficiently active, with a range of $26 \%$ to $85 \%$ not meeting the recommended physical activity levels. ${ }^{13}$ More recently, cross-sectional study reported that $82.6 \%$ of Saudi adults were not sufficiently active. ${ }^{14}$

Previous findings suggested a noteworthy and inverse association between the risk of MetS and meeting or exceeding the recommended MVPA level. ${ }^{15,16}$ For example, it has been reported that active people who met or exceeded the recommended 150 of MVPA per week had 36\% and 37\% lower risk of MetS, respectively. ${ }^{15}$ Furthermore, a metaanalysis found that physical activity reduced the incidence of MetS, in a dose-response manner. ${ }^{11}$ Light physical activity (LPA) refers to an activity that requires energy expenditure of 1.6-2.9 metabolic equivalent (MET), MVPA ( $\geq 3$ MET) and sedentary behavior $(\leq 1.5 \mathrm{MET})$ have been found significantly associated with MetS. ${ }^{16}$ A MET refers to the amount of oxygen consumed at rest and is equal to 3.5 (mL.kg.min ${ }^{-1}$ ). Data from Australia and Japan showed that LPA was associated with the reduction of MetS risks independently of MVPA and sedentary time. ${ }^{17,18}$ Another study from the United Kingdom (UK) suggested that conducting short bouts of LPA to break up sedentary time was associated with better metabolic outcomes, in older adults. ${ }^{19}$ Furthermore, there has been a strong correlation between sedentary behavior and the risk of MetS and its components. ${ }^{18,20}$ A study was conducted in the UK to investigate the effect of physical activity and sedentary time on metabolic health and liver adiposity. ${ }^{21}$ Results revealed that greater time spent in sedentary was associated with MetS, independent of physical activity levels. More recently, cross-sectional data suggested that high level of sedentary time was associated with poorer glycemic control in people with type 2 diabetes mellitus. ${ }^{22}$

However, the literature on this topic is mixed. For example, a study from Belgium found no association between LPA and MetS. ${ }^{23}$ These results were supported by the results of a Saudi study which found no association between LPA, MVPA, and sedentary time with MetS. ${ }^{24}$

The discrepancy in the association of physical activity and sedentary with MetS among previous studies is mainly due to the differences in sedentary and physical activity estimates. Different physical activity estimates could be attributed to different devices used, different data collecting and processing, participants' characteristics, and confounding variables. Objective measures such as accelerometers are widely used to estimate physical activity intensities and sedentary as an alternative of self-report methods which are limited by their variability and validity. $^{25}$ Many intensity thresholds (cut-points) have been developed from uniaxial and triaxial accelerometers to estimate physical activity levels and sedentary time. ${ }^{25}$ As a result, the relationship between physical activity and sedentary with various health outcomes might be influenced by the selected cut-points. ${ }^{26}$

Little is known about the associations of objectively measured physical activity and sedentary behavior with MetS in the Saudi community. The only study in Saudi Arabia reported that objectively measured physical activity and sedentary time using triaxial accelerometers did not predict MetS in males. ${ }^{24}$ However, this study had several limitations. For example, it was limited by including only young adults (mean age 37.6 years). Moreover, this study used cut-points were developed for uniaxial accelerometers. These cut-points may not be comparable with vector magnitude counts obtained from triaxial accelerometers. $^{25}$ Finally, the study did not take into account the independency of physical activity intensities and sedentary time in the association with MetS. More research is warranted to establish baseline data in order to identify effective interventions for preventing MetS in Saudi Community. To fill this gap, the present study aimed to examine whether objectively measured physical activity and sedentary time were independently associated with MetS in Saudi males from Riyadh city.

\section{Materials and Methods}

\section{Participants}

Participants from community development commission centers in Riyadh, Saudi Arabia, were requested to take part in this cross-sectional study. From the initial sample of 120 men (aged 33-78 years), 103 completed data and constituted the final sample. Eight participants were under 
40 years. Seventeen participants were excluded from data analysis because they did not meet the minimal wearing time of accelerometers which will be elaborated in the coming section.

To meet the inclusion criteria, all participants were required to be residents of Riyadh city, not having major cardiovascular (eg, heart disease, heart attack, stroke, arrhythmia, and heart failure) and musculoskeletal diseases, able to mobile independently, do not engage in professional sports, and to adhere to required wearing time of accelerometer. All measurements were performed in March and August 2018. All participants provided informed written consent. This study was conducted according to the principles outlined in the Declaration of Helsinki. The study protocol has been authorized by King Saud University's Institutional Review Board (IRB) (IRB No. E-18-3381).

\section{Instrumentation and Procedure}

All participant measurements were carried out by a professional laboratory technician in a clinical room. Height and weight were measured to the nearest $0.1 \mathrm{~cm}$ using a stadiometer (Seca 213, Seca GmbH \& Co., Hamburg, Germany), and to the nearest $0.1 \mathrm{~kg}$, using a digital scale (PD100 ProDoc, Detecto Scale, Cardinal, Webb City, MO, USA), respectively. From these measurements, their body mass index was calculated (BMI, in $\mathrm{kg}$ / $\mathrm{m}^{2}$ ). Waist circumference (WC) was measured at the umbilicus to the nearest $0.1 \mathrm{~cm}$ using a measuring tape. Resting heart rate (HRrest), systolic blood pressure (SBP), and diastolic blood pressure (DBP) readings ( $\mathrm{mmHg}$ ) were measured using an automatic arm digital sphygmomanometer (Omron HEM-7121, Omron Healthcare manufacturing, Japan). An average of three readings with an interval of 5-minutes rest in between was obtained while the participant sat on a chair with their arm supported at heart level.

A venous blood sample was collected after at least 10 hours of overnight fasting. Blood samples were analyzed to assess the level of HDL-C, TG, and FG. The National Cholesterol Education Program Adult Treatment Panel III guidelines (NCEP ATP III) modified by The American Heart Association (AHA) and the National Heart, Lung, and Blood Institute (NHLBI) ${ }^{27}$ were used to classify MetS in males. This was based on having three or more of the following: WC $(\geq 102)$, low HDL-C $(<1.03 \mathrm{mmol} / \mathrm{L})$, elevated TG $(\geq 1.7 \mathrm{mmol} / \mathrm{L})$, elevated blood glucose $(\geq 5.6$
mmol/L) and hypertension (SBP: $\geq 130 \mathrm{mmHg}$; DBP $\geq 85$ $\mathrm{mmHg}$ ).

Participants' physical activity levels and sedentary time were measured using an ActiGraph triaxial accelerometer (wGT3X-BT, ActiGraph LLC, Pensacola, FL). Devices were initialized, and then data were downloaded and analyzed using ActiLife v6013.3 (ActiGraph LLC, Pensacola, FL). At the first visit, all participants wore the accelerometers on their right hip using an elastic belt. Participants were asked to wear the accelerometers for seven consecutive days. Participants were instructed to always wear the accelerometer, except when they were bathing or during any other water-based activities such as swimming. At the final visit, all accelerometers were collected. The raw data were then downloaded per 10-seconds and reintegrated into 1-minute intervals for comparison with previous studies. Established accelerometer cut-points using vector magnitude (VM) were determined to categorize sedentary $^{28}$ and physical activity ${ }^{29}$ intensities as counts per minute (CPM): Sedentary: $<150$ CPM; LPA: 150 CPM - 2689 CPM; and MVPA $\geq 2690$ CPM.

\section{Data Reduction and Treatment}

The present data were managed in Microsoft Access 2016 and Microsoft Excel 2016. All completed accelerometer data were included in the final analysis. The algorithm from Troiano et $\mathrm{al}^{30}$ was used to compute valid accelerometer wear time. Only data collecting from 6 am until 11:59 pm were included in the analysis. ${ }^{31}$ Sustained 60 minutes of zero were classified as a period of non-wear time, with a tolerance of two minutes with non-zero values less than $100 \mathrm{CPM}^{30}$ Data were included in the analysis if participants wore the accelerometers for a minimum of four days, including one weekend day. ${ }^{25}$ A valid day required a minimum of 600 minutes of wear time. ${ }^{25}$ To examine the association of physical activity levels and sedentary with MetS, we categorized participants into tertiles of sedentary time $(\mathrm{T} 1>10.2$, T2 8.9-10.2, T3 $<8.9)$ LPA (T1 <5.5, T2 5.5-6.3, T3 >6.3) and MVPA (T1 <0.6, T2 0.6-0.8, T3 >0.8). In all models, T3 was included as a reference category. Time spent in MPA and VPA (min/day) for valid days were summed to calculate the average time spent in daily MVPA.

\section{Statistical Analysis}

Data were analyzed using SPSS (version 25, IBM). Continuous data were presented as a mean \pm standard deviation (SD) for variables with normal distribution and 
median (interquartile range IQR) for non-normal distributed variables. All continuous variables were checked for normality using the Kolmogorov-Smirnov test. If they were not normally distributed, then $\log$ transformation was applied. Categorical variables were presented as a frequency and percentage (\%). Our initial analysis revealed no significant differences between age groups (33-44, 45-59, and $\geq 60$ years) in the distribution of MetS $\left(\chi^{2}=0.467, P=0.792\right)$, mean of MVPA (F (2, $102)=0.175, P=0.260)$, LPA $(\mathrm{F}(2,102)=2.897, P=$ $0.334)$, and sedentary time $(\mathrm{F}(2,102)=3.074, P=0.428)$. Thus, in subsequent analysis, all age groups were analysed as a single group. An independent $t$-test was used to check the mean difference of variables with normal distribution. The Mann Whitney $U$-test was used to ascertain the median difference of non-normal distributed variables. A binary logistics regression analysis with simple enter method was performed to examine the association of sedentary and physical activity levels (independent variables) with MetS (dependent variable) (model 1). We created additional models 2, 3, and 4 which also mutually controlled for sedentary, light, and MVPA intensities. Data are presented as odds ratio and $95 \%$ confidence interval (CI). Variance inflation factors (VIF) were used to assess multicollinearity among independent variables. High multicollinearity was not detected as all values were $<10$. $^{32}$ A $p$-value of $<0.05$ was considered statistically significant.

\section{Results}

\section{Characteristics of the Study Sample}

Participants' characteristics and activities are presented in Table 1 . The data showed that $37.8 \%$ of the participants were found to have MetS. The data also showed that low HDL-C (51.4\%), FG (49.5\%), and hypertension (45.6\%) were the most prevalent MetS components, whereas WC (33\%) and elevated TG (38.8\%) were the lowest. All participants have normal range of DBP levels. Independent $t$-test showed no significant differences between participants with and without MetS in age and height (all $P>0.05$ ). Independent $t$-test results also showed that participants with MetS were heavier and have more BMI rates (all $P<0.05$ ).

\section{Differences in Metabolic Syndrome Components, Sedentary Time, and PA (Normal vs MetS)}

Differences in metabolic syndrome components, physical activities and sedentary behaviors are shown in Table 1.
The Mann Whitney $U$ and independent t-tests revealed that participants with MetS had significantly higher numbers of systolic BP, higher levels of glucose, higher WC, higher levels of triglycerides, and lower levels of HDL-C compared to those without MetS (all $P<0.05$ ). No significant difference was found between both groups in DBP (P > 0.05). Participants with and without MetS spent a similar amount of time in MVPA, sedentary bouts ( $\geq 30 \mathrm{~min}$ ), time wearing the accelerometer and number of bouts. Participants with MetS showed higher amounts of sedentary time and lower light intensity time. Median (IQR) daily accelerometer wear time was 16.7 (16.2-17.1) h/d.

\section{Regression Analysis Results}

Table 2 shows the binary logistic regression models and odds ratios (95\% CI) for associations between MetS and sedentary, LPA, and MVPA intensities. In unadjusted model 1 , we found that less time $(<5.5$ and $5.5-6.3 \mathrm{~h} / \mathrm{d})$ spent in the first and second tertiles of LPA intensity was significantly associated with higher odds of having MetS $(\mathrm{OR}=6.96$ and 4.64) compared with time more than 6.3$\mathrm{h} / \mathrm{d}$ in the referent tertile $(P$ trend $=0.001)$. Furthermore, less time spent in low levels of LPA intensity (first and second tertiles) was also significantly associated with higher odds of having MetS compared to time spent in higher level of LPA in the referent tertile when models controlled for sedentary time (model 2) and MVPA (model4) $(\mathrm{OR}=5.80-4.26 ; \mathrm{OR}=6.75-4.69$, respectively). The data showed no significant association between sedentary time and MetS across all models $\left(P_{\text {trend }}=0.082\right)$. The data also showed the association between MVPA and MetS was not statistically significant across all models $(P$ trend $=0.916)$.

\section{Discussion}

In the present study, we aimed to examine whether objectively measured physical activity and sedentary time were independently associated with MetS in 103 Saudi males from Riyadh city. Our main findings showed a statistically significant association of LPA with MetS independent of sedentary time and MVPA. In line with previous objectively measured physical activity studies, ${ }^{17-19}$ our study provides evidence for the role of LPA in reducing the risk of MetS among adults. We found that lower levels of LPA were associated with a higher risk of MetS, compared with the reference category (T3). For example, our data showed that less time than $6.3 \mathrm{~h} / \mathrm{d}$ spent in LPA was associated with significantly higher odds of having MetS across all 
Table I Clinical and Physical Activity Characteristics of Participants

\begin{tabular}{|c|c|c|c|}
\hline \multirow[t]{2}{*}{ Parameters } & \multirow[b]{2}{*}{ Normal } & \multirow[b]{2}{*}{ MetS } & \multirow[b]{2}{*}{$p$-value } \\
\hline & & & \\
\hline$N$ & $63( \pm 61.1 \%)$ & 39 (37.8\%) & \\
\hline Age (years) & $54.2( \pm 11.2)$ & $56(9.6)$ & 0.424 \\
\hline Height (cm) & $166.1( \pm 7.6)$ & $167.1(6.2)$ & 0.453 \\
\hline Weight $(\mathrm{Kg})$ & $75.8( \pm 12.9)$ & $85.1(11.5)^{* *}$ & $<0.001$ \\
\hline$W C(\mathrm{~cm})$ & $94(82-100)$ & $105(86-111) *$ & 0.002 \\
\hline BMI (kg/m2) & $27.1( \pm 3.8)$ & $30.6(3.8)^{* *}$ & $<0.001$ \\
\hline $\mathrm{SBP}(\mathrm{mmHg})$ & $125.1( \pm 19.1)$ & $134.4(16.9)^{*}$ & 0.014 \\
\hline $\mathrm{DBP}(\mathrm{mmHg})$ & $75.8( \pm 12.0)$ & $77.8(9.6)$ & 0.390 \\
\hline FBG (mmol/L) & $5.2(4.9-6.4)$ & $6.4(5.3-11.0)^{*}$ & 0.002 \\
\hline $\mathrm{HDL}-\mathrm{C}(\mathrm{mmol} / \mathrm{L})$ & I.I $( \pm 0.2)$ & $0.87(0.2)^{* *}$ & $<0.001$ \\
\hline Triglycerides (mmol/L) & I.I (0.9-1.4) & $2.1(1.7-2.9)$ & $<0.001$ \\
\hline MVPA $\left(h \cdot d^{-1}\right)$ & $0.7( \pm 0.3)$ & $0.6(0.2)$ & 0.334 \\
\hline LPA $\left(h . d^{-1}\right)$ & $6.2( \pm I . I)$ & $5.6(1.0)^{*}$ & 0.004 \\
\hline Sedentary $\left(h . d^{-1}\right)$ & $9.4( \pm 1.3)$ & $10.0(1.4)^{*}$ & 0.027 \\
\hline Sedentary Bouts; $\geq 30 \min \left(h \cdot d^{-1}\right)$ & 4.I(3.3-4.9) & $4.5(3.5-5.7)$ & 0.129 \\
\hline No. of Sedentary Bouts & $2.6(4-5.6)$ & $5.2(4.3-6.6)$ & 0.100 \\
\hline Accelerometer Wear Time (h.d ${ }^{-1}$ ) & $16.7(16.1-17.3)$ & $16.6(15.4-17.3)$ & 0.719 \\
\hline
\end{tabular}

Notes: Data are presented as mean $( \pm S D)$ and median $(I Q R) . *(P<0.05)$ and $* *(P<0.0 I)$ are considered significant.

Abbreviations: MetS, metabolic syndrome; WC, waist circumference; BMI, body mass index; SBP, systolic blood pressure; DBP, diastolic blood pressure; FBG, fasting glucose; HDL-C, high-density lipoprotein; MVPA, moderate-vigorous physical activity; LPA, light physical activity.

models $(\mathrm{OR}=4.26-6.96)$. This finding supports a recent meta-analysis of the association between objectively measured physical activity and all-cause mortality in adults. ${ }^{33}$ In their review, Ekelund et $\mathrm{al}^{33}$ suggested that maximal risk reductions in all-cause mortality were reported when engaging in LPA for 375 minutes per day $(6.25 \mathrm{~h} / \mathrm{d})$. This amount of LPA is similar to the amount of the highest tertile $(6.3 \mathrm{~h} / \mathrm{d})$ in our study. Our findings were also similar to those of other previous studies, ${ }^{17,18}$ which found that low levels of LPA were significantly associated with an increased risk of having MetS. However, our data was not in agreement with all previous works. A Saudi study ${ }^{24}$ found no association between time spent in LPA and the prevalence of MetS in Saudi males. Similarly, studies from China $^{9}$ and Belgium ${ }^{23}$ found no association between time spent in LPA and the prevalence of MetS in males and females. Inequivalent finding with previous studies may be attributed to the discrepancy in the prevalence of MetS reported in the current study compared to previous studies. Additionally, we used a triaxial accelerometer to assess physical activity intensities, while previous studies have used a self-report method or uniaxial accelerometer.

Our results confirm that the association between LPA with MetS remained significant after models were also controlled for sedentary and MVPA. This finding is consistent with the results of previous studies. ${ }^{17-19}$ This suggests that LPA has an important role in reducing the risk of MetS and should be used for public health promotion. This suggestion is also supported by the results of a previous study, which reported that LPA was associated with reducing mortality risk $^{8,34}$ and cardiometabolic health ${ }^{34}$ in adults. However, as age increases, it is challenging for some adults to practice and maintain long bouts of MVPA. ${ }^{19}$ We found that older participants spent less time in long bouts of MVPA by 9.5 minutes (data are not presented). Therefore, increasing the daily levels of LPA could be a practical way of preventing or reducing MetS in middle-aged and older Saudi males. In agreement with previous suggestions, ${ }^{18}$ we also suggest that physical activity recommendations should be updated to include LPA. As a greater daily time of LPA may be needed to prevent MetS, ${ }^{18}$ we found that time over $6.3 \mathrm{~h} / \mathrm{d}$ spent in LPA may reduce the risk of MetS. Further studies are needed to determine the lower limit of LPA that can reduce the incidence of MetS.

The association between sedentary behavior and MetS has become a growing research area. Therefore, the effect of sedentary behavior on the prevalence of MetS was also investigated in the present study. We did not find a similar statistically significant association between sedentary time and MetS in the current study as those found in previous 


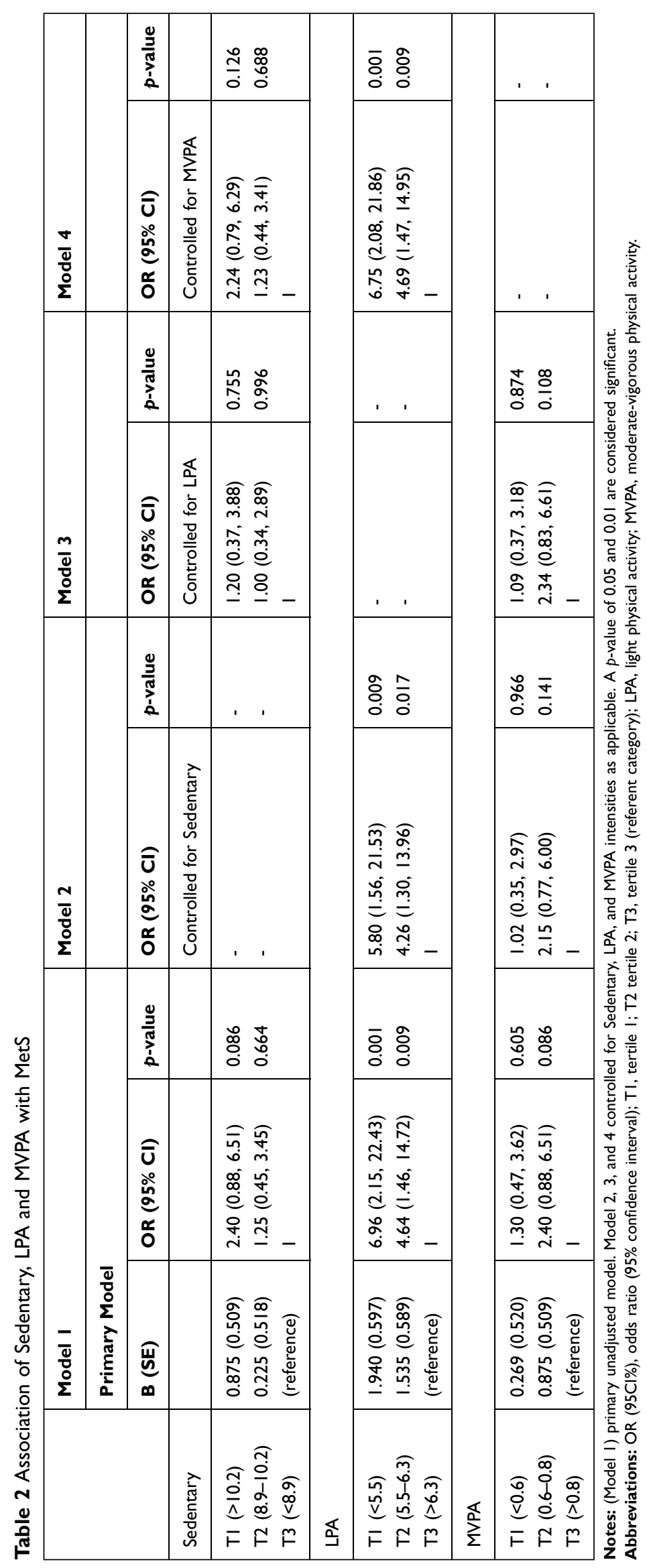


studies. ${ }^{17,18}$ However, our data reported that more time per day spent in sedentary behavior was associated with higher risk of having MetS. The results also indicate that the odds ratio for MetS was higher, but not statistically significant when models controlled for MVPA and LPA. Our findings are similar to the results found in previous study in Saudi males. ${ }^{24}$ Further research with larger sample sizes and adjustment for potential covariates such as dietary habit, smoking, and income is warranted to confirm these results in the Saudi population. Our results, alongside previous studies, may support the opinion that meeting the recommended physical activity may not be sufficient if sedentary time is not reduced. ${ }^{35}$ It has been reported that sedentary behavior could be an independent determent of health risk. ${ }^{20}$ Observing the different types of sedentary behavior was not one of our study aims. However, time spent watching television and using a computer has been found to be significantly associated with MetS independent of MVPA. ${ }^{36,37}$ Initiatives to increase the population's physical activity levels should also aim to reduce sedentary time. ${ }^{38}$

The positive effect of MVPA in reducing the risk of MetS is well documented. ${ }^{19}$ However, our results are in agreement with the previous Saudi study, which found no association between MVPA levels and MetS. ${ }^{24}$ The weak associations found in this study compared to previous studies could be owing to the low amount of MVPA observed. One possible contributing factor may be relating to the cut-points used, which was originally developed for adults to determine time spent in MVPA. Using these cut-points with older adults (who compromise a small proportion of the present sample) may underestimate their time spent in MVPA. However, it is not correct to use multiple cut-points in a single sample. Our findings support a previous meta-analysis, which reported a weak association between MVPA and reduction of MetS after additional adjustment for sedentary time. ${ }^{39}$ The percentage of overweight and obese participants in our study was $79 \%$. Thus, another reason might be that overweight and obese participants could not accumulate sufficient time in MVPA. A previous cross-sectional study found that MetS was not associated with meeting physical activity recommendations in obese people unless $\geq 42$ minutes of moderate or $\geq 21$ minutes of vigorous physical activity per day were accumulated. ${ }^{40}$ Direct comparison to prior studies is challenging as some have different ways of measuring physical activity, different population characteristics (ie, age and gender) and did not take into account some potential confounders (ie, diet and smoking status). Together, this may cause a bias in our results.

\section{Strength and Limitations}

Our cross-sectional study has a strength that we objectively measured physical activity levels and sedentary time using a triaxial accelerometer. Thus, our physical activity and sedentary time data are less prone to biases compared to self-reported data. However, using different data collection and processing methods could be problematic for interpreting the association between physical activity with MetS. Our study also has several limitations. The design of this study was cross-sectional, so the causality of the direction between physical activity and MetS could not be inferred. We only included wake time data; therefore, another limitation was that sleep time was excluded from the data collection and processing. Current evidence suggests that sleep duration and sleep disorders may negatively affect cardiometabolic health outcomes. $^{41}$ The sample of the current study only included males, so the direction of the association between physical activity or sedentary time and MetS in females is unknown. In the present study, several confounders such as calorie intake, smoking, family history of a metabolic disorder, and physical fitness, which may influence the association between physical activity and MetS were not controlled for. Self-reported comorbidities including health information about used medication were not collected from participants. Self-reported comorbidities data may have an effect in the associations between MetS and physical activity and sedentary time. Future studies should take this tool in consideration when examining the associations between MetS and physical activity and sedentary time. Finally, the study was carried out on a small sample of males from Riyadh, Saudi Arabia. It is therefore not possible to confirm that these findings are representative of the wider male population residing in Saudi Arabia. Further studies are required using larger samples of males from multiple Saudi Arabia regions.

\section{Conclusions}

This study found a significant association between LPA and MetS, independent of MVPA and sedentary time. For public health promotion, our results suggest that increasing LPA should be considered to reduce the prevalence of MetS in males from Riyadh city. Longitudinal studies are needed to investigate the efficacy of LPA in preventing 
and treating MetS before concrete recommendations can be made.

\section{Acknowledgments}

The authors are highly grateful to all Community Development Commissions of Riyadh districts who contributed to the study by contacting the older population of the community and hosting data collection. We also thank Research Support and Services Unit at Deanship of Scientific Research at KSU for the technical support.

\section{Author Contributions}

All authors contributed to data analysis, drafting or revising the article, have agreed on the journal to which the article will be submitted, gave final approval of the version to be published, and agree to be accountable for all aspects of the work.

\section{Funding}

The study has received a grant through Research Group Program (RG-1439-82) from the Deanship of Scientific Research at King Saud University (KSU), Riyadh, Saudi Arabia.

\section{Disclosure}

The authors declare no conflicts of interest for this work.

\section{References}

1. Alexander CM, Landsman PB, Teutsch SM, Haffner SM. NCEP-defined metabolic syndrome, diabetes, and prevalence of coronary heart disease among NHANES III participants age 50 years and older. Diabetes. 2003;52(5):1210-1214. doi:10.2337/diabetes.52.5.1210

2. Grundy SM, Brewer HB Jr, Cleeman JI, Smith SC Jr, Lenfant C. Definition of metabolic syndrome: report of the National Heart, Lung, and Blood Institute/American Heart Association conference on scientific issues related to definition. Arterioscler Thromb Vasc Biol. 2004;24(2):e13-e18. doi:10.1161/01.ATV.0000111245.75752.C6

3. Alberti KG, Eckel RH, Grundy SM, et al. Harmonizing the metabolic syndrome: a joint interim statement of the International Diabetes Federation Task Force on Epidemiology and Prevention; National Heart, Lung, and Blood Institute; American Heart Association; World Heart Federation; International Atherosclerosis Society; and International Association for the Study of Obesity. Circulation. 2009;120 (16):1640-1645. doi:10.1161/CIRCULATIONAHA.109.192644

4. Al-Rubeaan K, Bawazeer N, Al Farsi Y, et al. Prevalence of metabolic syndrome in Saudi Arabia - a cross sectional study. BMC Endocr Disord. 2018;18(1):16. doi:10.1186/s12902-018-0244-4

5. Wahid A, Manek N, Nichols M, et al. Quantifying the association between physical activity and cardiovascular disease and diabetes: a systematic review and meta-analysis. J Am Heart Assoc. 2016;5(9). doi:10.1161/JAHA.115.002495.

6. Smith AD, Crippa A, Woodcock J, Brage S. Physical activity and incident type 2 diabetes mellitus: a systematic review and dose-response meta-analysis of prospective cohort studies. Diabetologia. 2016;59(12):2527-2545. doi:10.1007/s00125-0164079-0
7. Liu X, Zhang D, Liu Y, et al. Dose-response association between physical activity and incident hypertension: a systematic review and meta-analysis of cohort studies. Hypertension. 2017;69(5):813-820. doi:10.1161/HYPERTENSIONAHA.116.08994

8. Saint-Maurice PF, Troiano RP, Berrigan D, Kraus WE, Matthews CE. Volume of light versus moderate-to-vigorous physical activity: similar benefits for all-cause mortality? J Am Heart Assoc. 2018;7(7). doi:10.1161/JAHA.118.008528

9. Xiao J, Shen C, Chu MJ, et al. Physical activity and sedentary behavior associated with components of metabolic syndrome among people in rural China. PLoS One. 2016;11(1):e0147062. doi:10.1371/ journal.pone. 0147062

10. Zhang WH, Xue P, Yao MY, Chang HM, Wu Y, Zhang L. Prevalence of metabolic syndrome and its relationship with physical activity in suburban Beijing, China. Ann Nutr Metab. 2013;63(4):298-304. doi:10.1159/000355575

11. Zhang D, Liu X, Liu Y, et al. Leisure-time physical activity and incident metabolic syndrome: a systematic review and dose-response meta-analysis of cohort studies. Metabolism. 2017;75:36-44. doi:10.1016/j.metabol.2017.08.001

12. Physical Activity Guidelines Advisory Committee. 2018 Physical Activity Guidelines Advisory Committee Scientific Report. Washington D.C., USA: U.S. Department of Health and Human Services; 2018.

13. Al-Hazzaa HM. Physical inactivity in Saudi Arabia revisited: a systematic review of inactivity prevalence and perceived barriers to active living. Int $J$ Health Sci (Qassim). 2018;12(6):50-64.

14. Alqahtani BA, Alenazi AM, Alhowimel AS, Elnaggar RK. The descriptive pattern of physical activity in Saudi Arabia: analysis of national survey data. Int Health. 2020. doi:10.1093/inthealth/ihaa027

15. Wu S, Fisher-Hoch SP, Reininger B, McCormick JB. Recommended levels of physical activity are associated with reduced risk of the metabolic syndrome in Mexican-Americans. PLoS One. 2016;11(4): e0152896. doi:10.1371/journal.pone.0152896

16. Kim YJ, Hwang JY, Kim H, Park S, Kwon O. Diet quality, physical activity, and their association with metabolic syndrome in Korean adults. Nutrition. 2019;59:138-144. doi:10.1016/j.nut.2018.08.009

17. Mitchell BL, Smith AE, Rowlands AV, Parfitt G, Dollman J. Associations of physical activity and sedentary behaviour with metabolic syndrome in rural Australian adults. J Sci Med Sport. 2018;21 (12):1232-1237. doi:10.1016/j.jsams.2018.05.002

18. Kim J, Tanabe K, Yokoyama N, Zempo H, Kuno S. Objectively measured light-intensity lifestyle activity and sedentary time are independently associated with metabolic syndrome: a cross-sectional study of Japanese adults. Int J Behav Nutr Phys Act. 2013;10:30. doi:10.1186/1479-5868-10-30

19. Jefferis BJ, Parsons TJ, Sartini C, et al. Does duration of physical activity bouts matter for adiposity and metabolic syndrome? A cross-sectional study of older British men. Int J Behav Nutr Phys Act. 2016;13(1):36. doi:10.1186/s12966-016-0361-2

20. Edwardson CL, Gorely T, Davies MJ, et al. Association of sedentary behaviour with metabolic syndrome: a meta-analysis. PLoS One. 2012;7(4):e34916. doi:10.1371/journal.pone.0034916

21. Bowden Davies KA, Sprung VS, Norman JA, et al. Physical activity and sedentary time: association with metabolic health and liver fat. Med Sci Sports Exerc. 2019;51(6):1169-1177. doi:10.1249/ MSS.0000000000001901

22. Alothman S, Alshehri MM, Alenazi AM, et al. The association between sedentary behavior and health variables in people with type 2 diabetes. Health Beh Policy Issue. 2020;7(3):198-206.

23. Scheers T, Philippaerts R, Lefevre J. SenseWear-determined physical activity and sedentary behavior and metabolic syndrome. Med Sci Sports Exerc. 2013;45(3):481-489. doi:10.1249/MSS.0b013e31827563ba

24. Alkahtani S, Elkilany A, Alhariri M. Association between sedentary and physical activity patterns and risk factors of metabolic syndrome in Saudi men: a cross-sectional study. BMC Public Health. 2015;15:1234. doi:10.1186/s12889-015-2578-4 
25. Migueles JH, Cadenas-Sanchez C, Ekelund U, et al. Accelerometer data collection and processing criteria to assess physical activity and other outcomes: a systematic review and practical considerations. Sports Med (Auckland, N Z). 2017;47(9):1821-1845. doi:10.1007/ s40279-017-0716-0

26. Loprinzi PD, Lee H, Cardinal BJ, Crespo CJ, Andersen RE, Smit E. The relationship of actigraph accelerometer cut-points for estimating physical activity with selected health outcomes: results from NHANES 2003-06. Res $Q$ Exerc Sport. 2012;83(3):422-430. doi:10.1080/02701367.2012.10599877

27. Grundy SM, Cleeman JI, Daniels SR, et al. Diagnosis and management of the metabolic syndrome: an American Heart Association/ National Heart, Lung, and Blood Institute Scientific Statement. Circulation. 2005;112(17):2735-2752. doi:10.1161/CIRCULATION AHA.105.169404

28. Peterson NE, Sirard JR, Kulbok PA, DeBoer MD, Erickson JM. Validation of accelerometer thresholds and inclinometry for measurement of sedentary behavior in young adult university students. Res Nurs Health. 2015;38(6):492-499. doi:10.1002/nur.21694

29. Sasaki JE, John D, Freedson PS. Validation and comparison of ActiGraph activity monitors. J Sci Med Sport. 2011;14(5):411-416. doi:10.1016/j.jsams.2011.04.003

30. Troiano RP, Berrigan D, Dodd KW, Masse LC, Tilert T, McDowell M. Physical activity in the United States measured by accelerometer. Med Sci Sports Exerc. 2008;40(1):181-188. doi:10.1249/mss.0b013e31815a51b3

31. Zisko N, Nauman J, Sandbakk SB, et al. Absolute and relative accelerometer thresholds for determining the association between physical activity and metabolic syndrome in the older adults: the Generation-100 study. BMC Geriatr. 2017;17(1):109. doi:10.1186/ s12877-017-0497-1

32. O'brien RM. A caution regarding rules of thumb for variance inflation factors. Qual Quant. 2007;41:673-690. doi:10.1007/s11135-0069018-6

33. Ekelund U, Tarp J, Steene-Johannessen J, et al. Dose-response associations between accelerometry measured physical activity and sedentary time and all cause mortality: systematic review and harmonised meta-analysis. BMJ. 2019;366:14570. doi:10.1136/bmj.14570
34. Chastin SFM, De Craemer M, De Cocker K, et al. How does light-intensity physical activity associate with adult cardiometabolic health and mortality? Systematic review with meta-analysis of experimental and observational studies. Br J Sports Med. 2019;53 (6):370-376. doi:10.1136/bjsports-2017-097563

35. González K, Fuentes J, Márquez JL. Physical inactivity, sedentary behavior and chronic diseases. Korean J Fam Med. 2017;38 (3):111-115. doi:10.4082/kjfm.2017.38.3.111

36. Ford ES, Kohl HW III, Mokdad AH, Ajani UA. Sedentary behavior, physical activity, and the metabolic syndrome among US adults. Obes Res. 2005;13(3):608-614. doi:10.1038/oby.2005.65

37. Bertrais S, Beyeme-Ondoua JP, Czernichow S, Galan P, Hercberg S, Oppert JM. Sedentary behaviors, physical activity, and metabolic syndrome in middle-aged French subjects. Obes Res. 2005;13 (5):936-944. doi:10.1038/oby.2005.108

38. Leiva AM, Martinez MA, Cristi-Montero C, et al. [Sedentary lifestyle is associated with metabolic and cardiovascular risk factors independent of physical activity]. Rev Med Chil. 2017;145 (4):458-467. doi:10.4067/S0034-98872017000400006

39. He D, Xi B, Xue J, Huai P, Zhang M, Li J. Association between leisure time physical activity and metabolic syndrome: a meta-analysis of prospective cohort studies. Endocrine. 2014;46 (2):231-240. doi:10.1007/s12020-013-0110-0

40. Xu F, Cohen SA, Lofgren IE, Greene G, Delmonico MJ, Greaney ML. The association between physical activity and metabolic syndrome in older adults with obesity. J Frailty Aging. 2019;8(1):27-32.

41. St-Onge M-P, Grandner MA, Brown D, et al. Sleep duration and quality: impact on lifestyle behaviors and cardiometabolic health: a scientific statement from the American Heart Association. Circulation. 2016;134(18):e367-e386. doi:10.1161/CIR.0000000 000000444
Risk Management and Healthcare Policy

\section{Publish your work in this journal}

Risk Management and Healthcare Policy is an international, peerreviewed, open access journal focusing on all aspects of public health, policy, and preventative measures to promote good health and improve morbidity and mortality in the population. The journal welcomes submitted papers covering original research, basic science, clinical \& epidemiological studies, reviews and evaluations, guidelines, expert opinion and commentary, case reports and extended reports. The manuscript management system is completely online and includes a very quick and fair peer-review system, which is all easy to use. Visit http://www.dovepress.com/testimonials.php to read real quotes from published authors. 